Article

\title{
Anthelmintic Activity of Wormwood (Artemisia absinthium L.) and Mallow (Malva sylvestris L.) against Haemonchus contortus in Sheep
}

\author{
Dominika Mravčáková ${ }^{1}$, Michaela Komáromyová ${ }^{2}$, Michal Babják $^{2}$, Michaela Urda Dolinská $^{2}$, \\ Alžbeta Königová ${ }^{2}$, Daniel Petrič ${ }^{1}$, Klaudia Čobanová ${ }^{1}$, Sylwester Ślusarczyk ${ }^{3}$, \\ Adam Cieslak ${ }^{4}$, Marián Várady ${ }^{2, *}$ and Zora Váradyová ${ }^{1, *}$ \\ 1 Institute of Animal Physiology, Centre of Biosciences of Slovak Academy of Sciences, 04001 Košice, Slovakia; \\ mravcakova@saske.sk (D.M.); petric@saske.sk (D.P.); boldik@saske.sk (K.Č.) \\ 2 Institute of Parasitology of Slovak Academy of Sciences, 04001 Košice, Slovakia; \\ komaromyova@saske.sk (M.K.); babjak@saske.sk (M.B.); dolinska@saske.sk (M.U.D.); konig@saske.sk (A.K.) \\ 3 Department of Pharmaceutical Biology with Botanical Garden of Medicinal Plants, Medical University of \\ Wroclaw, 50-556 Wroclaw, Poland; sylwester.slusarczyk@umed.wroc.pl \\ 4 Department of Animal Nutrition, Poznan University of Life Sciences, 60-637 Poznan, Poland; \\ adam.cieslak@up.poznan.pl \\ * Correspondence: varady@saske.sk (M.V.); varadyz@saske.sk (Z.V.); Tel.: +421-55-633-1411-13 (M.V.); \\ +421-55-792-2972 (Z.V.)
}

Received: 8 December 2019; Accepted: 26 January 2020; Published: 29 January 2020

Simple Summary: The gastrointestinal parasitic nematode Haemonchus contortus of small ruminants is an important target for chemoprophylaxis. Repeated use of anthelmintics in the form of synthetic drugs increases the risk of residues in food products and the development of anthelmintic resistance. However, the use of combinations of dry traditional medicinal plants as nutraceuticals is an alternative to chemotherapeutics for controlling haemonchosis in ruminants. Therefore, the aim of this study is to determine the effect of dietary supplementation with wormwood, mallow and their mix on parasitological status and inflammatory response in lambs experimentally infected with $H$. contortus. Simultaneously, the present study evaluated by the egg hatch test the in vitro anthelminthic effects of different concentrations $(50-1.563 \mathrm{mg} / \mathrm{mL}$ ) of the aqueous extracts of these plants. Our results revealed that the strong anthelmintic effect of both medicinal plants observed in vitro was not fully confirmed in vivo. This knowledge builds on our previously published findings and highlights that the effect of dry medicinal plants depends on the variety and synergy of plant polyphenols and the combination of bioactive compounds that together have an effect and contribute to a certain pharmacological efficacy.

Abstract: The objective of this study is to evaluate the effect of dry wormwood and mallow on the gastrointestinal parasite of small ruminants Haemonchus contortus. Twenty-four experimentally infected lambs were randomly divided into four groups of six animals each: unsupplemented lambs, lambs supplemented with wormwood, lambs supplemented with mallow and animals supplemented with a mix of both plants. Faecal samples from the lambs were collected on day 23, 29, 36, 43, 50, 57,64 and 75 post-infection for quantification of the number of eggs per gram (EPG). The mix of both plants contained phenolic acids (10.7 g/ $/ \mathrm{kg} \mathrm{DM})$ and flavonoids ( $5.51 \mathrm{~g} / \mathrm{kg} \mathrm{DM})$. The nematode eggs were collected and in vitro egg hatch test was performed. The aqueous extracts of both plants exhibited strong ovicidal effect on $H$. contortus, with ED50 and ED99 values of 1.40 and $3.76 \mathrm{mg} / \mathrm{mL}$ and 2.17 and $5.89 \mathrm{mg} / \mathrm{mL}$, respectively, in the in vitro tests. Despite the great individual differences between the treated lambs in eggs reduction, the mean EPG of the untreated and treated groups did not differ $(p>0.05)$. Our results indicate that using wormwood and mallow as dietary supplements do not have a sufficient effect on lambs infected with $H$. contortus. 
Keywords: dietary treatments; plant bioactive compounds; egg counts; UHRMS; Haemonchus contortus

\section{Introduction}

The gastrointestinal nematode (GIN) infection haemonchosis is a prevalent parasitic disease associated with economic losses, lowered productivity, morbidity and mortality. Haemonchus contortus is highly prevalent in sheep and goats worldwide and is mainly controlled by chemoprophylaxis through the repeated application of chemotherapeutics with the risk of development of anthelmintic resistance [1].

The screening of traditional medicinal plants containing promising contents of bioactive compounds with anthelmintic activity has great potential as an alternative source of natural anthelmintics and antioxidants that may be sustainable and environmentally acceptable. Various bioactive compounds (i.e., polyphenols, flavonoids, condensed tannins) that possess an anthelmintic effect $[2,3]$ and antibacterial and antioxidant activities have been isolated from wormwood (Artemisia absinthium L.) [4,5]. Many authors have reported the antioxidant and antimicrobial properties of wormwood essential oils [6,7] and the anthelmintic activity of the flavonoids quercetin and apigenin [3]. Diets containing dried wormwood as a $5 \%-10 \%$ replacement for rice straw also provide better quality roughage with a considerable content of crude protein [8-10]. The high pharmacological activity of the medicinal plant mallow (Malva sylvestris L.), due to the presence of amino acids, flavonoids, mucilages, terpenoids, phenol derivatives, enzymes, coumarins and sterols, is known [11,12]. Mallow has antimicrobial, antifungal and anti-inflammatory properties [13,14].

In traditional medicine, whole plants or mixtures of plants are used rather than isolated compounds, and therefore more research is needed on all types of interaction between plant constituents. The ultra-high-resolution mass spectrometry (UHRMS) analyses of dry medicinal plants or plant mixtures in our recent studies with $H$. contortus [15-17] identified a wide range of bioactive compounds with important pharmacological activities, mainly flavonoids, phenolic acids, diterpenes, and alkaloids. These experiments, which combined chromatographic analyses with the determination of antioxidant capacity, are helpful in identifying plants with consistent concentrations of anthelmintic and antioxidant compounds for in vitro and in vivo studies. Our previous studies showed that medicinal plant mixtures are multicomponent mixes that possess effects via a multitarget additive and synergistic mode [16-18].

Therefore, in the present study, we hypothesize that some medicinal plants from these mixtures are by themselves multicomponent mixes and can elicit effects via pharmacological activity on $H$. contortus infected lambs. The medicinal plants, wormwood and mallow, were chosen based on their previously described best phytotherapeutic properties and anthelmintic activity in vitro [15]. The goal is to determine the effect of dietary supplementation with wormwood and mallow on parasitological status and inflammatory parameters of lambs experimentally infected with $H$. contortus.

\section{Material and Methods}

\subsection{Ethics Statement}

All procedures and animals were cared for under European Community guidelines (EU Directive 2010/63/EU). The experimental protocol was approved by the Ethical Committee of the Institute of Parasitology of the Slovak Academy of Sciences, in accordance with national legislation in Slovakia. 


\subsection{Analysis of Bioactive Compounds}

Wormwood and mallow were ground to a fine powder, and $100 \mathrm{mg}$ were extracted three times in $80 \% \mathrm{MeOH}$ for $30 \mathrm{~min}$ at $40{ }^{\circ} \mathrm{C}$. The extracts were evaporated to dryness, dissolved in $2 \mathrm{~mL}$ of Milli-Q water (acidified with $0.2 \%$ formic acid) and purified by Solid Phase Extraction (SPE) using Oasis HLB 3cc Vac Cartrige (60 mg, Waters Corp., Milford, MA, USA). The cartridges were washed with $0.5 \%$ methanol to remove carbohydrates and then washed with $80 \%$ methanol to elute phenolics. The phenolic fraction was re-evaporated and dissolved in $1 \mathrm{~mL}$ of $80 \%$ methanol (acidified with $0.1 \%$ formic acid). The sample was then centrifuged $(23,000 \times g, 5 \mathrm{~min})$ before spectrometric analysis. All analyses were performed in triplicate for three independent samples and stored in a freezer at $-20^{\circ} \mathrm{C}$ before analysis. The phenolic acids and flavonoids of the plant materials were analysed by a ultra-high-resolution mass spectrometry (Dionex UltiMate 3000RS, Thermo Scientific, Darmstadt, Germany) system with a charged aerosol detector interfaced with a high-resolution quadrupole time-of-flight mass spectrometer (HR/Q-TOF/MS, Compact, Bruker Daltonik GmbH, Bremen, Germany). The metabolomes of the samples were chromatographically separated, as was described [19]. The flow rate, spectra, operating parameters, collision energy, data calibration and spectra processing were previously described [20]. The amount of the particular phenolic acids in the samples was calculated as the chlorogenic acid (CAS 327-97-9, 3-Caffeoylquinic acid) equivalent, and hyperoside (CAS 482-36-0, quercetin 3-galactoside) was used for calculating the amount of identified flavonoids. Stock solutions of hyperoside and chlorogenic acids were prepared in $\mathrm{MeOH}$, as was described previously [16].

\subsection{In Vitro Test}

The in vitro egg hatch test (EHT) was performed in order to assess the ovicidal effect of aqueous extracts of wormwood and mallow and compared with the chemotherapeutic effect of thiabendazole anthelmintic drug. The nematode eggs for in vitro EHT were obtained from the untreated UNS group. The concentrations of aqueous extracts used and EHT has been previously described [15].

Chemical tests for the screening of main constituents in the medicinal plants under study were carried out in the aqueous extracts using standard procedures [21,22]. Qualitative phytochemical screening revealed the active compounds mainly tannins, flavonoids, glycosides, saponins, alkaloids, and terpenoids (Table 1).

Table 1. Chemical composition of the aqueous plant extracts.

\begin{tabular}{ccccccc}
\hline Plant Species & Tannins & Flavonoids & Glycosides & Saponins & Alkaloids & Terpenoids \\
\hline A. absinthium & + & - & - & + & - & + \\
M. sylvestris & + & + & + & - & + & + \\
\hline
\end{tabular}

(+): the presence of phytochemicals; (-): the absence of phytochemicals.

\subsection{Experiment In Vivo}

The experiment was conducted on 24 3-4-month-old female lambs (Improved Valachian) with initial body weights of $18.67 \pm 0.55 \mathrm{~kg}$. The lambs were housed in common stalls on a sheep farm (Hodkovce, Slovak Republic) with free access to water. After a period of adaptation, all parasite-free lambs were infected by L3 larvae of $H$. contortus MHCo1 strain [16]. The diet of each animal consisted of oats (500 g DM/d) and meadow hay (ad libitum). Four groups of six animals based on their live-weight were established: unsupplemented lambs (UNS), lambs supplemented with stem of A. absinthium (ART, $1 \mathrm{~g} \mathrm{DM} / \mathrm{d} / \mathrm{lamb}$ ), lambs supplemented with flower of M. sylvestris (MAL, $15 \mathrm{~g} \mathrm{DM} / \mathrm{d} / \mathrm{lamb}$ ) and animals supplemented with mix of $A$. absinthium and $M$. sylvestris (ARTMAL, $16 \mathrm{~g} \mathrm{DM} / \mathrm{d} / \mathrm{lamb}$ ). The doses of plant supplements were based on the plant proportions used in our previous study [18]. The dry plants from commercial sources (AGROKARPATY, Plavnica, Slovak Republic) were mixed daily with the oats during the experimental period (75 days, D). The lambs were weighed on D0, D15, D30, D45 and D70. Faecal samples from the rectum of lambs were collected on D23, D29, D36, D43, D50, D57, 
D64 and D75 post-infection for quantification of the eggs per gram (EPG). The detection of strongylid eggs was performed by McMaster technique, as was previously described [23]. The blood sera samples of each animal were obtained from D15, D30, D45 and D70 [18]. Helminthological autopsy were done after 75 days of infection [16].

\subsection{Blood Sera Analysis}

Sheep immunoglobulin G (IgG), sheep immunoglobulin A (IgA) and sheep eosinophil peroxidase (EPX) were measured by ELISA kits (MyBioSource Ltd., San Diego, CA, USA). The sensitivity of the IgG, IgA and EPX kits were $0.938 \mathrm{ng} / \mathrm{mL}, 1.875 \mathrm{ng} / \mathrm{mL}$ and $1.0 \mathrm{ng} / \mathrm{mL}$, respectively.

\subsection{Statistical Analysis}

Statistical analysis was performed using analysis variance (GraphPad Prism 8, GraphPad Software, Inc., San Diego, CA, USA) as repeated-measures mixed models representing the four animal groups (UNS, ART, MAL, ARTMAL) and sampling days. Differences between the animal groups were analysed by a two-way ANOVA with a Bonferroni post hoc test. Differences between the arithmetic EPG means between groups and between worm counts at dissection were analysed by Student's $t$-tests. A logistic regression model was used to determine the $\mathrm{ED}_{50}$ and $\mathrm{ED}_{99}$ [24].

\section{Results}

\subsection{Bioactive Compounds}

The A. absinthium contained $6.48 \mathrm{~g} / \mathrm{kg}$ DM of phenolic acids and $0.35 \mathrm{~g} / \mathrm{kg}$ DM of flavonoids with greater concentrations of chlorogenic acid $(3.42 \mathrm{~g} / \mathrm{kg} \mathrm{DM})$ and 1,5-dicaffeoylquinic acid $(2.12 \mathrm{~g} / \mathrm{kg}$ DM) (Table 2). The M. sylvestris contained $0.65 \mathrm{~g} / \mathrm{kg}$ DM of phenolic acids and $6.48 \mathrm{~g} / \mathrm{kg} \mathrm{DM}$ of flavonoids with higher concentrations of delphinidin-5-glucoside 3-lathyroside (1.64 g/ $\mathrm{kg} \mathrm{DM})$, kaempferol-3-O-rutinoside $(0.82 \mathrm{~g} / \mathrm{kg} \mathrm{DM})$, apigenin-o-hex $(1.56 \mathrm{~g} / \mathrm{kg} \mathrm{DM})$ and coumarinic acid $(0.47 \mathrm{~g} / \mathrm{kg}$ DM). The mix of both plants contained $10.7 \mathrm{~g} / \mathrm{kg}$ DM phenolic acids and $5.51 \mathrm{~g} / \mathrm{kg}$ DM of flavonoids with greater concentrations of methyl-4-O-beta-d-glucopyranosylcaffeate ( $2.23 \mathrm{~g} / \mathrm{kg} \mathrm{DM})$, 1,5-dicaffeoylquinic acid (1.64 g/kg DM), kaempferol-O-Hex (1.40 g/kg DM), apigenin-O-Hex (1.29 g/kg $\mathrm{DM})$ and luteolin-O-Hex $(0.70 \mathrm{~g} / \mathrm{kg} \mathrm{DM})$. 
Table 2. Contents of the flavonoids and phenolic acids $(\mathrm{g} / \mathrm{kg} \mathrm{DM})$ identified in the plants and mix.

\begin{tabular}{|c|c|c|c|c|c|c|c|c|c|}
\hline No. & RT (min) & $\lambda_{\max }(\mathrm{nm})$ & $\begin{array}{c}m / z \\
{[\mathrm{M}-\mathrm{H}]^{-}}\end{array}$ & MS $^{2}$ & MS $^{2}$ Fragments & Formula & Compound & Flavonoids & Phenolic Acids \\
\hline \multicolumn{10}{|c|}{ Artemisia absinthium } \\
\hline 1 & 2.80 & & 189.0759 & $127 / 0759$ & $171 / 145 / 115$ & $\mathrm{C}_{8} \mathrm{H}_{14} \mathrm{O}_{5}$ & L-(-)Malic acid diethyl ester & & 0.22 \\
\hline 2 & 4.10 & 215.325 & 353.0877 & $191 / 0567$ & $179 / 161 / 135$ & $\mathrm{C}_{16} \mathrm{H}_{18} \mathrm{O}_{9}$ & Chlorogenic acid & & 3.42 \\
\hline 3 & 7.80 & & 281.1023 & & & $\mathrm{C}_{14} \mathrm{H}_{18} \mathrm{O}_{6}$ & ND & 0.01 & \\
\hline 4 & 8.00 & & 367.1031 & $191 / 0546$ & 173 & $\mathrm{C}_{17} \mathrm{H}_{20} \mathrm{O}_{9}$ & 3-O-Feruloylquinic acid & & 0.08 \\
\hline 5 & 8.90 & & 279.1223 & $234 / 1009$ & $261 / 217 / 177 / 199$ & $\mathrm{C}_{15} \mathrm{H}_{20} \mathrm{O}_{5}$ & Artabsinolide & & \\
\hline 6 & 9.10 & & 325.1283 & 163 & $279 / 235$ & $\mathrm{C}_{16} \mathrm{H}_{22} \mathrm{O}_{7}$ & ND & & 0.03 \\
\hline 7 & 9.20 & & 327.1440 & 279 & 235 & $\mathrm{C}_{16} \mathrm{H}_{24} \mathrm{O}_{7}$ & ND & & \\
\hline 8 & 10.00 & 289.000 & 263.1282 & $201 / 1271$ & $245 / 219 / 149 / 161 / 177$ & $\mathrm{C}_{15} \mathrm{H}_{20} \mathrm{O}_{4}$ & Tanacetin & & \\
\hline 9 & 10.20 & & 281.1386 & $219 / 373$ & $263 / 237 / 201$ & $\mathrm{C}_{16} \mathrm{H}_{24} \mathrm{O}_{7}$ & Artabsinolide D & & \\
\hline 10 & 11.00 & & 515.1193 & $353 / 0867$ & $191 / 179 / 135$ & $\mathrm{C}_{25} \mathrm{H}_{24} \mathrm{O}_{12}$ & 1,5-Dicaffeoylquinic acid & & 2.12 \\
\hline 11 & 11.20 & & 653.1719 & $345 / 0595$ & $330 / 302$ & $\mathrm{C}_{29} \mathrm{H}_{34} \mathrm{O}_{17}$ & Spinacetin 3-rutinoside & 0.24 & \\
\hline 12 & 11.40 & & 477.1032 & $314 / 0415$ & 357 & $\mathrm{C}_{22} \mathrm{H}_{22} \mathrm{O}_{12}$ & Isorhamnetin 7-glucoside & 0.10 & \\
\hline 13 & 11.70 & & 515.1192 & $353 / 0869$ & 173/179/191/155 & $\mathrm{C}_{25} \mathrm{H}_{24} \mathrm{O}_{12}$ & 4,5-Dicaffeoylquinic acid & & 0.61 \\
\hline 14 & 14.90 & & 507.1502 & $413 / 1246$ & $101 / 324 / 259$ & $\mathrm{C}_{24} \mathrm{H}_{28} \mathrm{O}_{12}$ & Hedycoryside B & & \\
\hline 15 & 15.00 & & 511.2698 & $467 / 2775$ & 405 & $\mathrm{C}_{30} \mathrm{H}_{40} \mathrm{O}_{7}$ & Anabsin & & \\
\hline 16 & 15.50 & & 345.1344 & $301 / 1433$ & 257/213/187 & $\mathrm{C}_{19} \mathrm{H}_{22} \mathrm{O}_{6}$ & Diosbulbin E & & \\
\hline 17 & 15.60 & & 511.2698 & $245 / 1175$ & $263 / 201$ & $\mathrm{C}_{30} \mathrm{H}_{40} \mathrm{O}_{7}$ & Anabsin & & \\
\hline \multirow[t]{3}{*}{18} & 16.50 & & 329.2323 & $211 / 1324$ & 229/171/183/139 & $\mathrm{C}_{18} \mathrm{H}_{34} \mathrm{O}_{5}$ & Pinellic acid & & \\
\hline & & & \multicolumn{5}{|c|}{ Total flavonoids and phenolic acids } & 0.35 & 6.48 \\
\hline & & & & & & Malva sylvestris & & & \\
\hline 1 & 1.60 & 250.320 & 517.1195 & $355 / 0667$ & 193 & $\mathrm{C}_{21} \mathrm{H}_{26} \mathrm{O}_{15}$ & Ferullo-O-Hex-O-Hex & & 0.02 \\
\hline 2 & 1.80 & 250.301 & 206.0443 & $144 / 0437$ & & $\mathrm{C}_{10} \mathrm{H}_{9} \mathrm{NO}_{4}$ & ND & & 0.17 \\
\hline 3 & 7.00 & 523 & 757.1846 & $347 / 0761$ & $329 / 261 / 509$ & $\mathrm{C}_{32} \mathrm{H}_{39} \mathrm{O}_{21}$ & Delphinidin 5-glucoside 3-lathyroside & 1.64 & \\
\hline 4 & 7.90 & 308 & 163.0381 & $119 / 0502$ & & $\mathrm{C}_{9} \mathrm{H}_{8} \mathrm{O}_{3}$ & Coumaric acid & & 0.47 \\
\hline 5 & 8.00 & 288 & 465.1046 & $303 / 0505$ & 285/275/177 & $\mathrm{C}_{21} \mathrm{H}_{22} \mathrm{O}_{12}$ & Xeractinol & 0.17 & \\
\hline 6 & 8.20 & 520 & 449.1094 & $287 / 0555$ & $259 / 243$ & $\mathrm{C}_{21} \mathrm{H}_{22} \mathrm{O}_{11}$ & Cyanidin-O-Hex & 0.28 & \\
\hline 7 & 8.50 & 518 & 593.1645 & $431 / 0982$ & $269 / 0460$ & $\mathrm{C}_{27} \mathrm{H}_{31} \mathrm{O}_{15}$ & Pelargonidin-O-Hex-O-Hex & 0.14 & \\
\hline 8 & 8.70 & 283 & 687.1784 & $507 / 1142$ & $345 / 0629 / 165$ & & ND & 0.18 & \\
\hline 9 & 9.00 & 283 & 525.1246 & $345 / 0815$ & $165 / 197 / 139$ & $\mathrm{C}_{23} \mathrm{H}_{25} \mathrm{O}_{14}$ & ND & 0.07 & \\
\hline 10 & 9.20 & 287 & 303.0498 & $153 / 0169$ & $125 / 217$ & $\mathrm{C}_{15} \mathrm{H}_{12} \mathrm{O}_{7}$ & ND & 0.04 & \\
\hline 11 & 9.50 & 283 & 773.1781 & $507 / 1124$ & $345 / 165$ & $\mathrm{C}_{32} \mathrm{H}_{38} \mathrm{O}_{22}$ & ND & 0.22 & \\
\hline 12 & 10.00 & & 609.1458 & $301 / 0330$ & & $\mathrm{C}_{27} \mathrm{H}_{31} \mathrm{O}_{16}$ & Quercetin-3-O-rutinoside & 0.40 & \\
\hline
\end{tabular}


Table 2. Cont

\begin{tabular}{|c|c|c|c|c|c|c|c|c|c|}
\hline No. & RT (min) & $\lambda_{\max }(\mathrm{nm})$ & $\begin{array}{c}m / z \\
{[\mathrm{M}-\mathrm{H}]^{-}}\end{array}$ & MS $^{2}$ & MS $^{2}$ Fragments & Formula & Compound & Flavonoids & Phenolic Acids \\
\hline 13 & 10.20 & 268.343 & 447.0928 & $285 / 0386$ & & $\mathrm{C}_{21} \mathrm{H}_{20} \mathrm{O}_{11}$ & Kaempferol-O-Hex & 0.49 & \\
\hline 14 & 10.50 & 346 & 505.0981 & $343 / 0442$ & & $\mathrm{C}_{23} \mathrm{H}_{22} \mathrm{O}_{13}$ & Quercetin 3'-glucoside-7-acetate & 0.03 & \\
\hline 15 & 10.90 & 266.343 & 593.1504 & $285 / 0395$ & & $\mathrm{C}_{27} \mathrm{H}_{30} \mathrm{O}_{15}$ & Kaempferol-3-O-rutinoside & 0.82 & \\
\hline 16 & 11.10 & 291.346 & 433.1124 & $271 / 0599$ & 151 & $\mathrm{C}_{21} \mathrm{H}_{22} \mathrm{O}_{10}$ & Naringenin-O-Hex & 0.13 & \\
\hline 17 & 11.25 & 291 & 287.0550 & $259 / 0596$ & $152 / 201 / 243$ & $\mathrm{C}_{15} \mathrm{H}_{12} \mathrm{O}_{6}$ & Tetrahydroxyflavone & 0.30 & \\
\hline 18 & 11.40 & 268.336 & 431.0978 & $269 / 0435$ & & $\mathrm{C}_{21} \mathrm{H}_{20} \mathrm{O}_{10}$ & Apigenin-O-Hex & 1.56 & \\
\hline 19 & 15.00 & 285.340 & 271.0595 & $151 / 0012$ & $177 / 119$ & $\mathrm{C}_{15} \mathrm{H}_{12} \mathrm{O}_{5}$ & Naringenin & 0.01 & \\
\hline 20 & 15.40 & & 327.2169 & & & $\mathrm{C}_{18} \mathrm{H}_{32} \mathrm{O}_{5}$ & $\begin{array}{c}\text { (E)-10-(8-Hydroxyoctanoyloxy)-enoic } \\
\text { acid }\end{array}$ & & \\
\hline \multirow[t]{2}{*}{21} & 15.70 & 215.334 & 269.0443 & $151 / 0016$ & 225 & $\mathrm{C}_{15} \mathrm{H}_{10} \mathrm{O}_{5}$ & Trihydroxyflavone & 0.02 & \\
\hline & & & \multicolumn{5}{|c|}{ Total flavonoids and phenolic acids } & 6.50 & 0.66 \\
\hline \multicolumn{10}{|c|}{ Mix of $A$. absinthium and M. sylvestris } \\
\hline 1 & 4.00 & 215.325 & 353.0883 & $191 / 0561$ & $173 / 179$ & $\mathrm{C}_{16} \mathrm{H}_{18} \mathrm{O}_{9}$ & 4-O-Caffeoylquinic acid & & 0.61 \\
\hline 2 & 4.10 & 215.325 & 353.0883 & $191 / 0561$ & $179 / 173$ & $\mathrm{C}_{16} \mathrm{H}_{18} \mathrm{O}_{9}$ & 3-O-Caffeoylquinic acid & & 0.74 \\
\hline 3 & 5.90 & 215.287 & 355.1035 & $193 / 0498$ & $149 / 134$ & $\mathrm{C}_{16} \mathrm{H}_{20} \mathrm{O}_{9}$ & $\begin{array}{l}\text { 1-O-2'-Hydroxy-4'- } \\
\text { methoxycinnamoyl } \\
\text {-b-D-glucose }\end{array}$ & & 0.38 \\
\hline 4 & 6.10 & 215.302 & 355.1038 & $149 / 0598$ & 193/134 & $\mathrm{C}_{16} \mathrm{H}_{20} \mathrm{O}_{9}$ & 1-O-Feruloylglucose & & 0.70 \\
\hline 5 & 7.90 & & 161.0225 & $133 / 0282$ & & $\mathrm{C}_{9} \mathrm{H}_{6} \mathrm{O}_{3}$ & Umbeliferone & & 0.40 \\
\hline 6 & 8.00 & & 323.0760 & $161 / 0221$ & & $\mathrm{C}_{15} \mathrm{H}_{16} \mathrm{O}_{8}$ & Mahaleboside & & 0.02 \\
\hline 7 & 8.10 & 225.287 & 465.1033 & $303 / 177$ & $285 / 0399$ & $\mathrm{C}_{21} \mathrm{H}_{22} \mathrm{O}_{12}$ & Xeractinol & 0.04 & \\
\hline 8 & 8.30 & 520.000 & 449.1094 & $287 / 0555$ & $259 / 243$ & $\mathrm{C}_{21} \mathrm{H}_{22} \mathrm{O}_{11}$ & Cyanidin-O-Hex & 0.03 & \\
\hline 9 & 8.50 & & 367.1025 & $173 / 0433$ & $193 / 155 / 134$ & $\mathrm{C}_{17} \mathrm{H}_{20} \mathrm{O}_{9}$ & Feruloylquinic acid & & 0.25 \\
\hline 10 & 9.00 & 233.294 .318 & 355.1034 & 193/0507 & $149 / 134$ & $\mathrm{C}_{16} \mathrm{H}_{20} \mathrm{O}_{9}$ & $\begin{array}{l}\text { Methyl-4-O-beta-D- } \\
\text { glucopyranosylcaffeate }\end{array}$ & & 2.23 \\
\hline 11 & 9.80 & 255.354 & 463.0882 & $301 / 0337$ & 343 & $\mathrm{C}_{21} \mathrm{H}_{20} \mathrm{O}_{12}$ & Quercetin O-Hex & 0.44 & \\
\hline 12 & 9.90 & 252.351 & 609.1472 & $301 / 0331$ & $285 / 0415$ & $\mathrm{C}_{27} \mathrm{H}_{30} \mathrm{O}_{16}$ & Isoquercitrin O-Dhex & 0.42 & \\
\hline 13 & 10.30 & 257,4 & 447.0920 & $285 / 0386$ & & $\mathrm{C}_{21} \mathrm{H}_{20} \mathrm{O}_{11}$ & Kaempferol-O-Hex & 1.40 & \\
\hline 14 & 10.70 & 217.291 .325 & 515.1189 & $353 / 0877$ & $179 / 191$ & $\mathrm{C}_{25} \mathrm{H}_{24} \mathrm{O}_{12}$ & 3,5-Dicaffeoylquinic acid & & 0.80 \\
\hline 15 & 10.80 & & 187.0958 & $125 / 0968$ & 169 & $\mathrm{C}_{9} \mathrm{H}_{16} \mathrm{O}_{4}$ & ND & & \\
\hline 16 & 10.90 & 221.329 & 593.1520 & $285 / 0397$ & & $\mathrm{C}_{27} \mathrm{H}_{30} \mathrm{O}_{15}$ & Kaempferol-3-O-rutinoside & 0.37 & \\
\hline 17 & 11.10 & 217.291 .325 & 515.1197 & $353 / 0869$ & $191 / 179$ & $\mathrm{C}_{25} \mathrm{H}_{24} \mathrm{O}_{12}$ & 1,5-Dicaffeoylquinic acid & & 1.64 \\
\hline 18 & 11.15 & 291.346 & 433.1124 & $271 / 0599$ & 151 & $\mathrm{C}_{21} \mathrm{H}_{22} \mathrm{O}_{10}$ & Naringenin-O-Hex & 0.19 & \\
\hline
\end{tabular}


Table 2. Cont.

\begin{tabular}{|c|c|c|c|c|c|c|c|c|c|}
\hline No. & RT (min) & $\lambda_{\max }(\mathrm{nm})$ & $\begin{array}{c}m / z \\
{[\mathrm{M}-\mathrm{H}]^{-}}\end{array}$ & MS $^{2}$ & MS $^{2}$ Fragments & Formula & Compound & Flavonoids & Phenolic Acids \\
\hline 19 & 11.40 & 266.3 & 431.0976 & $269 / 0434$ & & $\mathrm{C}_{21} \mathrm{H}_{20} \mathrm{O}_{10}$ & Apigenin O-Hex & 0.56 & \\
\hline 20 & 11.50 & 268.343 & 447.0928 & $285 / 0386$ & & $\mathrm{C}_{21} \mathrm{H}_{20} \mathrm{O}_{11}$ & Luteolin O-Hex & 0.70 & \\
\hline 21 & 11.60 & 266.3 & 431.0976 & $269 / 0434$ & & $\mathrm{C}_{21} \mathrm{H}_{20} \mathrm{O}_{10}$ & Apigenin O-Hex & 0.73 & \\
\hline 22 & 11.70 & 215.290 .325 & 515.119 & $353 / 0868$ & $173 / 179 / 191$ & $\mathrm{C}_{25} \mathrm{H}_{24} \mathrm{O}_{12}$ & 4,5-Dicaffeoylquinic acid & & 0.68 \\
\hline 23 & 12.40 & 325.0 & 517.1342 & $355 / 1022$ & $353 / 193 / 149 / 161$ & $\mathrm{C}_{25} \mathrm{H}_{26} \mathrm{O}_{12}$ & 3-caffeoyl-4-dihydrocaffeoyl quinic acid & & 0.35 \\
\hline 24 & 12.90 & 268.320 & 639.3176 & $519 / 2604$ & $476 / 373 / 145$ & $\mathrm{C}_{37} \mathrm{H}_{44} \mathrm{~N}_{4} \mathrm{O}_{6}$ & Tris-trans-p-coumaroylspermine & & 0.50 \\
\hline 25 & 13.10 & 218.268 .339 & 473.1083 & $269 / 0426$ & 413 & $\mathrm{C}_{23} \mathrm{H}_{22} \mathrm{O}_{11}$ & Apigenin -O-(Hex-Ac) & 0.12 & \\
\hline 26 & 13.70 & 325.0 & 517.1330 & $323 / 0759$ & $353 / 193 / 149 / 161$ & $\mathrm{C}_{25} \mathrm{H}_{26} \mathrm{O}_{12}$ & 4-caffeoyl-3-dihydrocaffeoyl quinic acid & & 0.07 \\
\hline 27 & 14.20 & 218.268 .339 & 473.1083 & $269 / 0426$ & 413 & $\mathrm{C}_{23} \mathrm{H}_{22} \mathrm{O}_{11}$ & Apigenin -O-(Hex-Ac) & 0.22 & \\
\hline 28 & 14.40 & 266.336 & 515.1187 & $269 / 0444$ & & $\mathrm{C}_{25} \mathrm{H}_{24} \mathrm{O}_{12}$ & $\begin{array}{l}\text { Formononetin } \\
\text { 7-O-glucoside-6"-malonate }\end{array}$ & & 0.22 \\
\hline 29 & 15.00 & 285.340 & 271.0595 & $151 / 0012$ & $177 / 119$ & $\mathrm{C}_{15} \mathrm{H}_{12} \mathrm{O}_{5}$ & Naringenin & 0.07 & \\
\hline 30 & 15.40 & & 327.2169 & & & $\mathrm{C}_{18} \mathrm{H}_{32} \mathrm{O}_{5}$ & $\begin{array}{l}\text { (E)-10-(8-Hydroxyoctanoyloxy)dec-2-enoic } \\
\text { acid }\end{array}$ & 0.03 & \\
\hline 31 & 15.70 & 215.334 & 269.0443 & $151 / 0016$ & 225 & $\mathrm{C}_{15} \mathrm{H}_{10} \mathrm{O}_{5}$ & Trihydroxyflavone & 0.03 & \\
\hline 32 & 17.10 & 222.309 & 785.3554 & $545 / 2397$ & $665 / 502 / 399 / 145$ & $\mathrm{C}_{46} \mathrm{H}_{50} \mathrm{~N}_{4} \mathrm{O}_{8}$ & Tetra-trans-p-coumaroylspermine & & 0.47 \\
\hline 33 & 19.00 & & 373.0914 & $358 / 0681$ & $343 / 329 / 315$ & $\mathrm{C}_{19} \mathrm{H}_{18} \mathrm{O}_{8}$ & Dihydroxy-tetramethoxyflavone & 0.03 & \\
\hline 34 & 20.30 & 267.334 & 559.1069 & $269 / 0443$ & $515 / 1172$ & $\mathrm{C}_{26} \mathrm{H}_{24} \mathrm{O}_{14}$ & Apigenin 7-(2"-acyl-6"maloylglycosyl) & 0.13 & 0.65 \\
\hline & & & \multicolumn{5}{|c|}{ Total flavonoids and phenolic acids } & 5.51 & 10.7 \\
\hline
\end{tabular}

No: peak numbers from UV chromatograms; RT: retention time; $\lambda_{\max }$ : wavelengths of maximum absorption in the visible region; MS ${ }^{2}$ : main ion; ND: not determined. 


\subsection{In Vitro Test (EHT)}

The dose-response relationships of aqueous extracts of A. absinthium or M. sylvestris, respectively, against $H$. contortus in the egg hatch test (EHT) are shown in Figure 1a,b. Both aqueous plant extracts exhibited a strong ovicidal effect on $H$. contortus in in vitro EHT. The $\mathrm{ED}_{50}$ and $\mathrm{ED}_{99}$ values were 1.40 and $3.76 \mathrm{mg} / \mathrm{mL}$ in A. absinthium (Figure 1a) and 2.17 and $5.89 \mathrm{mg} / \mathrm{mL}$ in M. sylvestris (Figure 1b), respectively. Thiabendazole at a concentration of $1.0 \mu \mathrm{g} / \mathrm{mL}$ has a $100 \%$ ovicidal effect.
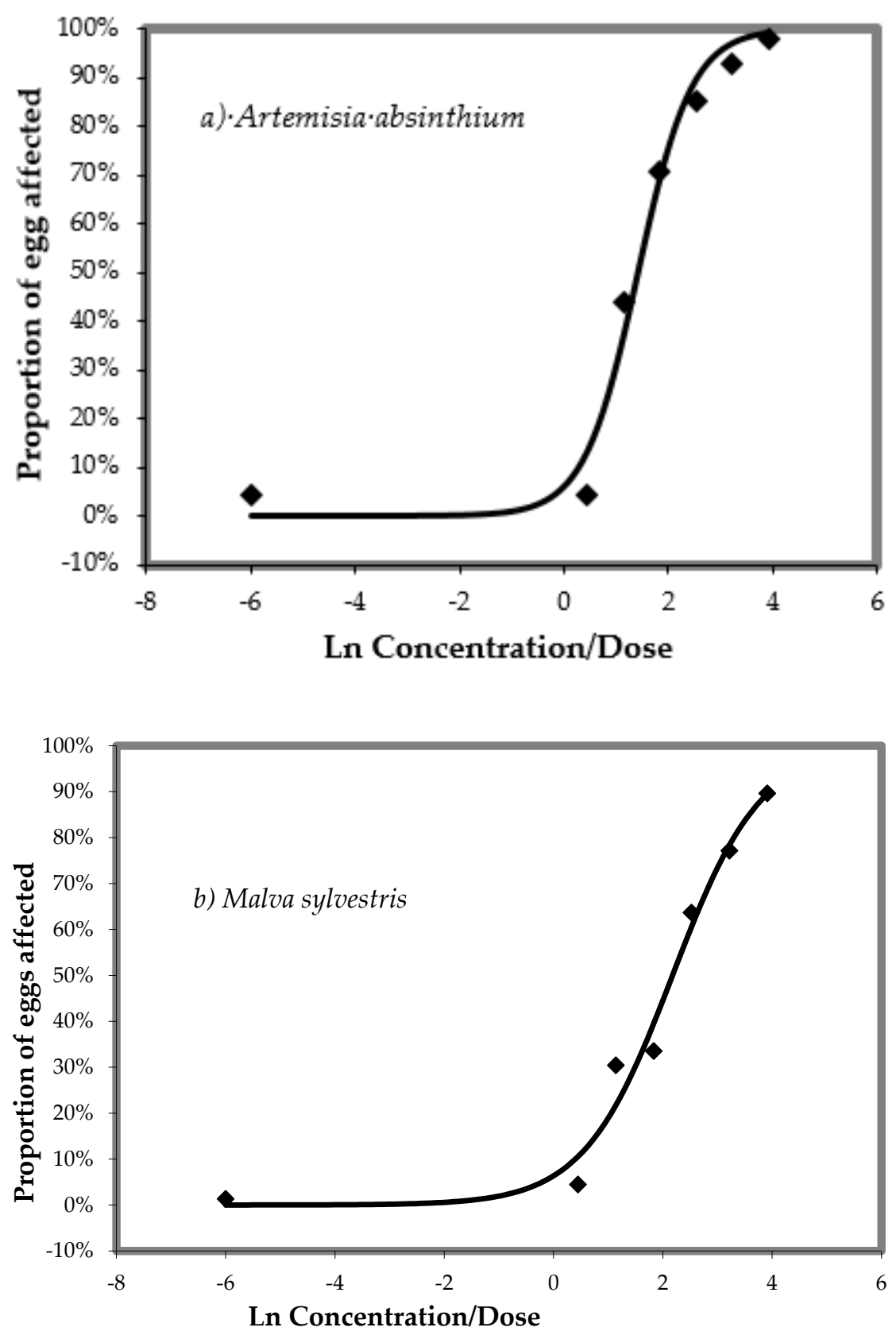

Figure 1. (a,b) Dose-response relationship of plant aqueous extracts against Haemonchus contortus in the egg hatch test (EHT) after $24 \mathrm{~h}$ of incubation at $26^{\circ} \mathrm{C}$.

\subsection{Parasitological Status}

The patterns of egg shedding for UNS, ART, MAL and ARTMAL are shown in Figure 2. Data from D36 were statistically compared and used to determine the reduction in egg output for ART, MAL and ARTMAL relative to UNS. Mean faecal eggs per gram (EPGs) were influenced by time from 
infection $(p<0.05)$, and for all groups, EPGs increased until D50 or D57, respectively. The EPGs in the lambs treated with MAL, ART and ARTMAL compared with UNS group did not differ $(p>0.05)$. The necropsy on D75 found a numerical decrease $(p>0.05)$ in the abomasal worm counts for the ARTMAL groups compared to the other groups (Figure 3).

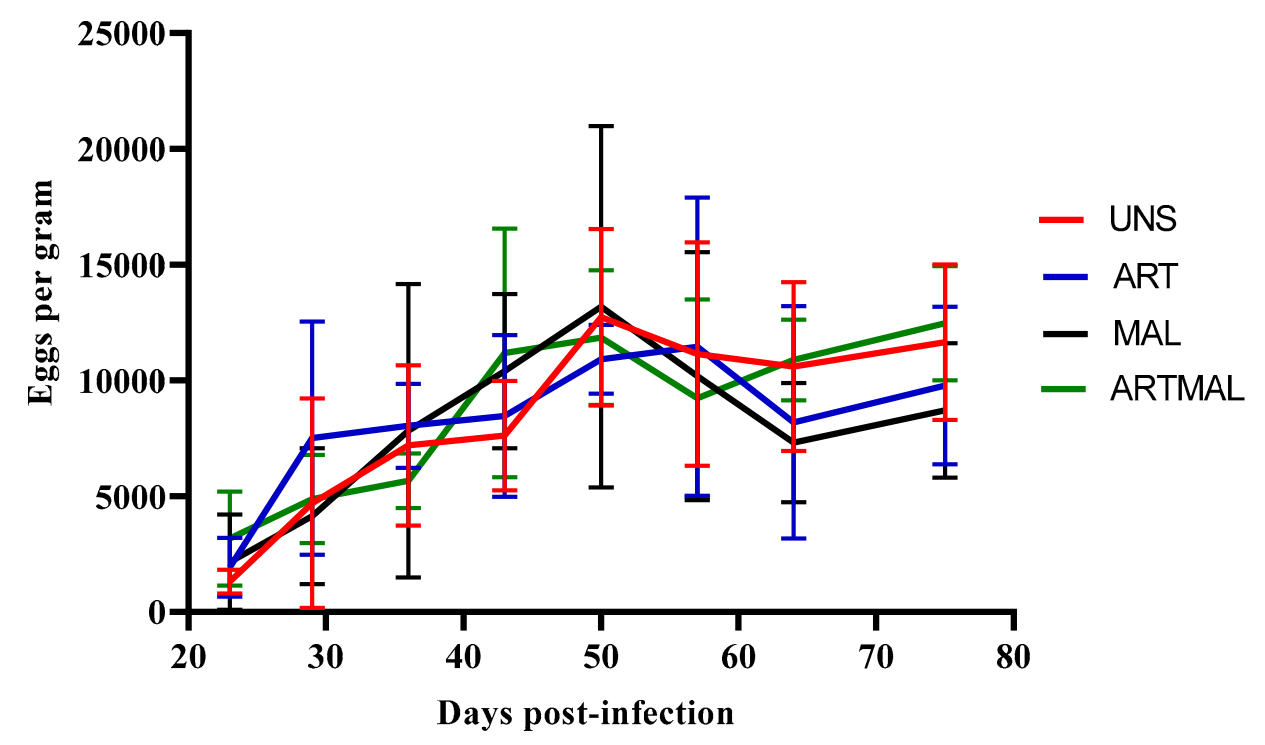

Figure 2. Mean faecal egg counts for the groups of lambs infected with Haemonchus contortus (Treatment: $p>0.05$; time: $p<0.001$; treatment $\times$ time: $p>0.05$ ). UNS: unsupplemented; ART: A. absinthium; MAL: M. sylvestris; ARTMAL: ART plus MAL.

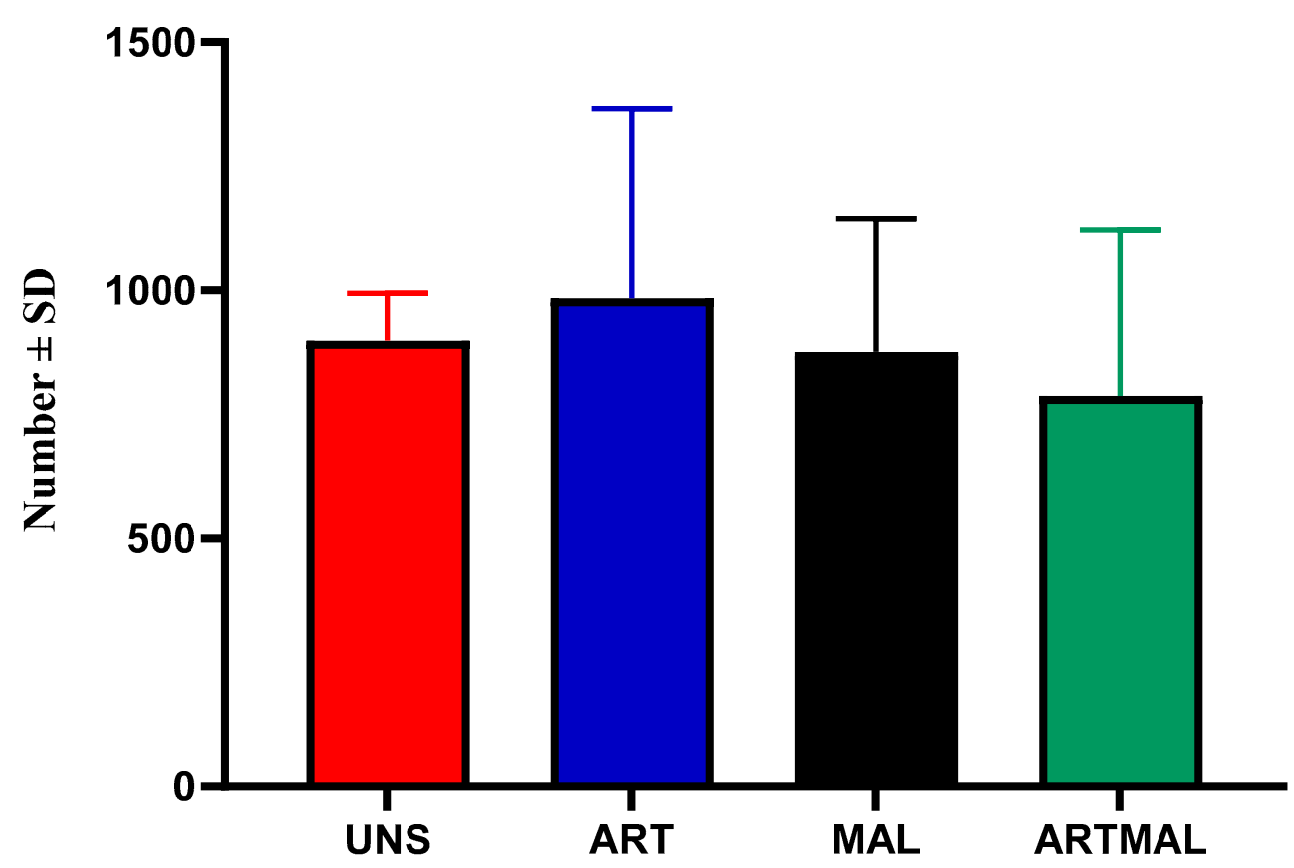

Figure 3. Abomasal worm counts of Haemonchus contortus in the lambs of each treatment at the end of the experiment $(p>0.05)$. UNS: unsupplemented; ART: A. absinthium; MAL: M. sylvestris; ARTMAL: ART plus MAL.

\subsection{Inflammatory Response}

Table 3 shows the inflammatory IgG, IgA and EPX response. Serum IgG and IgA values were not influenced by treatment, time and treatment $\times$ time $(p>0.05)$. Mean serum EPX values ranged in 
the treated groups from 24.1 to $73.4 \mathrm{ng} / \mathrm{mL}$, and the values were influenced by treatment and time $(p<0.05)$.

Table 3. Inflammatory responses of the experimental groups $(n=6)$.

\begin{tabular}{|c|c|c|c|c|c|c|c|c|c|}
\hline \multirow{2}{*}{ Item } & \multirow{2}{*}{ Day } & \multirow{2}{*}{ UNS } & \multirow{2}{*}{ ART } & \multirow{2}{*}{ MAL } & \multirow{2}{*}{ ARTMAL } & \multirow{2}{*}{ SD } & \multicolumn{3}{|c|}{ Significance of Effects } \\
\hline & & & & & & & Treatment (T) & Time & $\mathbf{T} \times$ Time \\
\hline \multirow{4}{*}{$\begin{array}{c}\operatorname{IgG} \\
(\mathrm{ng} / \mathrm{mL})\end{array}$} & 15 & 0.627 & 2.15 & 2.31 & 2.23 & 2.54 & \multirow{4}{*}{ NS } & \multirow{4}{*}{ NS } & \multirow{4}{*}{ NS } \\
\hline & 30 & 1.03 & 0.780 & 0.986 & 1.61 & 1.61 & & & \\
\hline & 45 & 1.33 & 0.639 & 0.378 & 1.04 & 1.07 & & & \\
\hline & 70 & 1.16 & 0.689 & 3.53 & 1.39 & 1.66 & & & \\
\hline \multirow{4}{*}{$\begin{array}{c}\operatorname{IgA} \\
(\mathrm{ng} / \mathrm{mL})\end{array}$} & 15 & 0.434 & 1.28 & 0.811 & 0.767 & 0.522 & \multirow{4}{*}{ NS } & \multirow{4}{*}{ NS } & \multirow{4}{*}{ NS } \\
\hline & 30 & 0.825 & 0.666 & 0.519 & 0.589 & 0.245 & & & \\
\hline & 45 & 0.529 & 0.438 & 0.787 & 0.485 & 0.233 & & & \\
\hline & 70 & 0.813 & 0.696 & 0.726 & 0.626 & 0.195 & & & \\
\hline \multirow{4}{*}{$\begin{array}{c}\text { EPX } \\
(\mathrm{ng} / \mathrm{mL})\end{array}$} & 15 & 37.5 & 66.6 & 52.2 & 50.0 & 17.9 & \multirow{4}{*}{ * } & \multirow{4}{*}{ * } & \multirow{4}{*}{ NS } \\
\hline & 30 & 44.0 & 36.9 & 29.4 & 44.8 & 13.9 & & & \\
\hline & 45 & 31.4 & 38.6 & 24.1 & 51.4 & 17.5 & & & \\
\hline & 70 & 49.2 & 33.4 & 37.8 & 73.4 & 21.1 & & & \\
\hline
\end{tabular}

UNS: unsupplemented; ART: A. absinthium; MAL: M. sylvestris; ARTMAL: ART plus MAL; EPX: eosinophil peroxidase; NS: not significant; SD: standard deviation. ${ }^{*} p<0.05$.

\section{Discussion}

In the present study, phenolic compounds, flavonoids and phenolic acids among them, were detected in wormwood, mallow and a mix of both plants. Phenolic acids, including chlorogenic acid, caffeoylquinic acid derivatives, coumaric acid and methyl 4-O-beta-D-glucopyranosylcaffeate, were identified in a range from 0.65 to $10.7 \mathrm{~g} / \mathrm{kg}$ DM. Chlorogenic acid and 1,5- and 4,5-dicaffeoylquinic acid possess well-known antibacterial, anthelmintic, anti-inflammatory, and antioxidant biological activities in vitro and in vivo [25,26]. Similarly, coumaric acid in Senegalia gaumeri leaf extract compounds has shown potential anthelmintic effects against $H$. contortus larvae [27]. The phenolic acid contents for wormwood and the mix (but not for mallow) were within the range of 3.6 to $57.3 \mathrm{~g} / \mathrm{kg} \mathrm{DM}$, as was reported for plant mixtures used previously in infected lambs [16,17]. In relation to flavonoids with antioxidant properties, we identified mainly flavones (apigenin and luteolin), flavonols (kaempferol and quercetin) and flavanones (naringenin) [28], which may also have anthelmintic activity [3,29]. However, the total content of flavonoids in wormwood in the present study was lower $(0.35 \mathrm{~g} / \mathrm{kg} \mathrm{DM})$ versus mallow or the mix ( 6.48 or $5.51 \mathrm{~g} / \mathrm{kg} \mathrm{DM}$, respectively) or compared to previous studies (9.96 and 29.5 or $41.5 \mathrm{~g} / \mathrm{kg}$ DM, respectively) [16,17].

It is clear that medicinal plants that have an anthelmintic effect in vitro are often not equally effective in vivo, because there is different bioavailability, pharmacology of host animals, metabolism of bioactive compounds by rumen microflora and experimental conditions [30]. In the present experiment, the aqueous plant extracts of both medicinal plants, A. absinthium and M. sylvestris, exhibited a strong ovicidal effect on $H$. contortus in vitro, similar to the extracts of the species Artemisia against sheep nematodes [31,32]. However, the mean egg outputs of the UNS group compared to ART, MAL and ARTMAL groups showed no significant differences in egg reduction in lambs. Egg production by $H$. contortus females remained high (i.e., thousands EPG) until D50 post-inoculation and then decreased similarly as during the patent period of $H$. contortus in sheep [33]. The rapid reduction in egg excretion after D50 (MAL and ARTMAL) or D57 (ART), respectively, was accompanied by a lower number (not statistically) of adult $H$. contortus worms in the ARTMAL group. No significant differences in egg excretion in the treated groups may have been due to the lower content of plant biologically active compounds, especially flavonoids, compared to our previous studies [16,17]. However, relatively high $\mathrm{SD}$ of the means of the treated groups in the present experiment point to a potentially different treatment effect between lambs. This suggests that these plant materials could have an indirect antiparasitic 
effect and may promote a host's resistance to parasitic infection in the longer term. However, the anthelmintic mechanism of action is unknown. It seems that flavonoids with antioxidant capacity, in particular, contribute to the indirect antiparasitic activity $[34,35]$. However, not only the content of flavonoids appears to play an important role in the anthelmintic activity of medicinal plants and their mixtures. Our results indicate that wormwood and mallow themselves are not responsible for egg and worm reduction in the lambs but probably acting in synergy with other medicinal plants and their bioactive compounds, as was done in previous mixes $[17,18]$. The effect of dry medicinal plants on the health of animals depends on the source of the multitarget complex bioactive compounds that work synergistically [36,37] and antagonistically [38]. An increase in the resistance of infected lambs to $H$. contortus infection was shown after the administration of mixtures of dry medicinal plants composed already of 9-13 species [16-18]. However, a generally great contribution to the discovery and development of new drugs is a widely applicable strategy for identifying the combinatory compounds responsible for a certain pharmacological activity of plant medicines followed by in vitro and in vivo validation [39].

Ruminal and intestinal fermentation parameters can be manipulated by supplementing a diet with medicinal plants [40]. No adverse effects of wormwood and mallow on the ruminal fermentation parameters (i.e., $\mathrm{pH}$, ammonia $\mathrm{N}$, methane, gas production, and volatile fatty acids) were found [18]. Mainly, $\mathrm{pH}$ and ammonia $\mathrm{N}$ can affect the release of phenolic compounds from plant materials and the growth of ruminal microbes for microbial protein synthesis [41,42]. Additionally, phenolic compounds, especially flavonoids, can improve body weight gain, growth and the quality of animal products [43]. In the present experiment, polyphenols as dietary supplements did not significantly affect the body weights or live-weight gains of the infected lambs. Dry medicinal plants in the diets of the infected lambs may [18] or may not have influenced the body weights or live-weight gains, which is consonant with a meta-analysis of gastro-intestinal nematode infection in sheep [44].

Our recent studies [16-18] of lambs infected with the gastrointestinal nematode $H$. contortus showed the potential value of medicinal plant mixtures to decrease egg output and worm numbers in parasitic infections of the digestive tract. However, this effect is probably not a consequence of a direct anthelmintic impact on the viability of nematodes, but an increase in the resistance of lambs to nematode infections. Additionally, a recent study also showed that a complementary vegetable mixture of plants belonging to the Compositae, Cesalpinacae, Liliacae, Bromeliaceae and Labiatae families used as feed at two different dosages was ineffective against gastrointestinal nematode infection [45]. It seems that mainly a combination of medicinal plants belonging to different botanical families with beneficial bioactive compounds probably contributes to slowing the dynamics of infection. In the present study, because of the low variety and synergy of plant polyphenols and the combination of bioactive compounds of wormwood and mallow, the reduction in parasitic infection intensity in the treated infected lambs was not sufficient during the 75 days of infection compared to previous studies [16-18]. However, it seems that mixtures of dry medicinal plants may affect the host over the longer term. Therefore, more research is needed on combinations of medicinal plants and interactions between compositions of plant mixtures for longer (90-120 days) experimental periods.

\section{Conclusions}

The data in the present study showed additional new knowledge on the anthelmintic effects of dry medicinal plants as dietary supplements. Our results indicate that using medicinal plants, even those with the best anthelmintic properties in vitro, may not have sufficient effects in vivo on $H$. contortus infected lamb.

Author Contributions: Conceptualization, Z.V., M.V.; Investigation, D.M., Z.V., M.V.; Supervision, M.V.; Formal analysis, D.M., M.K., K.Č., M.B., M.U.D., A.K., D.P., S.Ś.; Data Curation, Z.V., M.V., A.C.; Draft Preparation, Z.V.; Writing-Review \& Editing, Z.V., M.V. All authors have read and agreed to the published version of the manuscript.

Funding: This study was supported by funds from the Slovak Research and Development Agency (APVV 18-0131). 
Acknowledgments: The authors are grateful to Valéria Venglovská, Silvia Spišáková, Peter Jerga and Gabriel Benkovský for laboratory and technical assistance.

Conflicts of Interest: The authors declare no conflict of interest.

\section{References}

1. Lamb, J.; Elliott, T.; Chambers, M.; Chick, B. Broad spectrum anthelmintic resistance of Haemonchus contortus in Northern NSW of Australia. Vet. Parasitol. 2017, 24, 48-51. [CrossRef]

2. Tariq, K.A.; Chishti, M.Z.; Ahmad, F.; Shawl, A.S. Anthelmintic activity of extracts of Artemisia absinthium against ovine nematodes. Vet. Parasitol. 2009, 160, 83-88. [CrossRef]

3. Akkari, H.; Rtibi, K.; B'chir, F.; Rekik, M.; Darghouth, M.A.; Gharbi, M. In vitro evidence that the pastoral Artemisia campestris species exerts an anthelmintic effect on Haemonchus contortus from sheep. Vet. Res. Commun. 2014, 38, 249-255. [CrossRef]

4. Kordali, S.; Kotan, R.; Mavi, A.; Cakir, A.; Ala, A.; Yildirim, A. Determination of the chemical composition and antioxidant activity of the essential oil of Artemisia dracunculus and of the antifungal and antibacterial activities of Turkish Artemisia absinthium, A. dracunculus, Artemisia santonicum and Artemisia spicigera essential oils. J. Agric. Food Chem. 2005, 53, 9452-9458.

5. Kharoubi, O.; Slimani, M.; Krouf, D.; Seddik, L.; Aoues, A. Role of wormwood (Artemisia absinthium) extract on oxidative stress in ameliorating lead induced haematotoxicity. Afr. J. Tradit. Complement. Altern. Med. 2008, 5, 263-270. [CrossRef]

6. Msaada, K.; Salem, N.; Bachrouch, O.; Bousselmi, S.; Tammar, S.; Alfaify, A.; Al Sane, K.; Ammar, W.B.; Azeiz, S.; Brahim, A.H.; et al. Chemical Composition and Antioxidant and Antimicrobial Activities of Wormwood (Artemisia absinthium L.) Essential Oils and Phenolics. J. Chem. 2015, 804658. [CrossRef]

7. Nguyen, H.T.; Németh Zámboriné, É. Sources of variability of wormwood (Artemisia absinthium L.) essential oil. J. Appl. Res. Med. Aromat. Plants 2016, 3, 143-150. [CrossRef]

8. Kim, J.H.; Kim, C.H.; Ko, Y.D. Influence of dietary addition of dried wormwood (Artemisia sp.) on the performance and carcass characteristics of Hanwoo steers and the nutrient digestibility of sheep. Asian-Aust. J. Anim. Sci. 2002, 15, 390-395. [CrossRef]

9. Kim, Y.M.; Kim, J.H.; Kim, S.C.; Ha, H.M.; Ko, Y.D.; Kim, C.H. Influence of dietary addition of dried wormwood (Artemisia sp.) on the performance, carcass characteristics and fatty acid composition of muscle tissues of Hanwoo heifers. Asian-Aust. J. Anim. Sci. 2002, 15, 549-554. [CrossRef]

10. Ko, Y.D.; Kim, J.H.; Adesogan, A.T.; Ha, H.M.; Kim, S.C. The effect of replacing rice straw with dry wormwood (Artemisia sp.) on intake, digestibility, nitrogen balance and ruminal fermentation characteristics in sheep. Anim. Feed Sci. Technol. 2006, 125, 99-110. [CrossRef]

11. Cutillo, F.; D'Abrosca, B.; Dellagreca, M.; Fiorentino, A.; Zarrelli, A. Terpenoids and phenol derivatives from Malva silvestris. Phytochemistry 2006, 67, 481-485. [CrossRef] [PubMed]

12. Gasparetto, J.C.; Martins, C.A.; Hayashi, S.S.; Otuky, M.F.; Pontarolo, R. Ethnobotanical and scientific aspects of Malva sylvestris L.: A millennial herbal medicine. J. Pharm. Pharmacol. 2012, 64, 172-189. [CrossRef] [PubMed]

13. Zohra, S.F.; Meriem, B.; Samira, S. Some Extracts of Mallow Plant and its Role in Health. APCBEE Procedia 2013, 5, 546-550. [CrossRef]

14. Prudente, A.S.; Loddi, A.M.; Duarte, M.R.; Santos, A.R.; Pochapski, M.T.; Pizzolatti, M.G.; Hayashi, S.S.; Campos, F.R.; Pontarolo, R.; Santos, F.A.; et al. Pre-clinical anti-inflammatory aspects of a cuisine and medicinal millennial herb: Malva sylvestris L. Food Chem. Toxicol. 2013, 58, 324-331. [CrossRef]

15. Váradyová, Z.; Pisarčíková, J.; Babják, M.; Hodges, A.; Mravčáková, D.; Kišidayová, S.; Königová, A.; Vadlejch, J.; Várady, M. Ovicidal and larvicidal activity of extracts from medicinal-plants against Haemonchus contortus. Exp. Parasitol. 2018, 195, 71-77. [CrossRef]

16. Váradyová, Z.; Mravčáková, D.; Babják, M.; Bryszak, M.; Grešáková, L.; Čobanová, K.; Kišidayová, S.; Plachá, I.; Königová, A.; Cieslak, A.; et al. Effects of herbal nutraceuticals and/or zinc against Haemonchus contortus in lambs experimentally infected. BMC Vet. Res. 2018, 14, 78. [CrossRef]

17. Mravčáková, D.; Váradyová, Z.; Kopčáková, A.; Čobanová, K.; Grešáková, L.; Kišidayová, S.; Babják, M.; Urda Dolinská, M.; Dvorožňáková, E.; Königová, A.; et al. Natural chemotherapeutic alternatives for controlling of haemonchosis in sheep. BMC Vet. Res. 2019, 15, 302. [CrossRef] 
18. Váradyová, Z.; Kišidayová, S.; Čobanová, K.; Grešáková, L'.; Babják, M.; Königová, A.; Urda Dolinská, M.; Várady, M. The impact of a mixture of medicinal herbs on ruminal fermentation, parasitological status and hematological parameters of the lambs experimentally infected with Haemonchus contortus. Small Rumin. Res. 2017, 151, 124-132. [CrossRef]

19. Rodrigues, M.J.; Matkowski, A.; Ślusarczyk, S.; Magné, C.; Poleze, T.; Pereira, C.; Custódio, L. Sea knotgrass (Polygonum maritimum L.) as a potential source of innovative industrial products for skincare applications. Ind. Crop. Prod. 2019, 128, 391-398. [CrossRef]

20. Rodrigues, M.J.; Monteiro, I.; Placines, C.; Castañeda-Loaiza, V.; Ślusarczyk, S.; Matkowski, A.; Pereira, C.; Pousão-Ferreira, P.; Custódio, L. The irrigation salinity and harvesting affect the growth, chemical profile and biological activities of Polygonum maritimum L. Ind. Crop. Prod. 2019, 139, 111510. [CrossRef]

21. Yadav, R.N.S.; Agarwala, M. Phytochemical analysis of some medicinal plants. J. Phytol. 2011, 3, 10-14.

22. Jaradat, N.; Hussen, F.; Al Ali, A. Preliminary Phytochemical Screening, Quantitative Estimation of Total Flavonoids, Total Phenols and Antioxidant Activity of Ephedra alata Decne. J. Mater. Environ. Sci. 2015, 6, 1771-1778.

23. Coles, G.C.; Bauer, C.; Borgsteede, F.H.M.; Geerts, S.; Klei, T.R.; Taylor, M.A.; Waller, P.J. World Association for the Advancement of Veterinary Parasitology (W.A.A.V.P) methods for the detection of anthelmintic resistance in nematodes of veterinary importance. Vet. Parasitol. 1992, 44, 35-44. [CrossRef]

24. Babják, M.; Königová, A.; Urda Dolinská, M.; Vadlejch, J.; Várady, M. Anthelmintic resistance in goat herds-In vivo versus in vitro detection methods. Vet. Parasitol. 2018, 139, 10-14. [CrossRef] [PubMed]

25. Sato, Y.; Itagaki, S.; Kurokawa, T.; Ogura, J.; Kobayashi, M.; Hirano, T.; Sugawara, M.; Iseki, K. In vitro and in vivo antioxidant properties of chlorogenic acid and caffeic acid. Int. J. Pharm. 2011, 403, 136-138. [CrossRef] [PubMed]

26. Tajik, N.; Tajik, M.; Mack, I.; Enck, P. The potential effects of chlorogenic acid, the main phenolic components in coffee, on health: A comprehensive review of the literature. Eur. J. Nutr. 2017, 56, 2215-2244. [CrossRef] [PubMed]

27. Castañeda-Ramírez, G.S.; Torres-Acosta, J.F.J.; Sandoval-Castro, C.A.; Borges-Argáez, R.; Cáceres-Farfán, M.; Mancilla-Montelongo, G.; Mathieu, C. Bio-guided fractionation to identify Senegalia gaumeri leaf extract compounds with anthelmintic activity against Haemonchus contortus eggs and larvae. Vet. Parasitol. 2019, 270, 13-19. [CrossRef]

28. Kumar, S.; Pandey, A.K. Chemistry and biological activities of flavonoids: An overview. Sci. World J. 2013, 162750. [CrossRef]

29. Kozan, E.; Anul, S.A.; Tatli, I.I. In vitro anthelmintic effect of Vicia pannonica var. purpurascens on trichostrongylosis in sheep. Exp. Parasitol. 2013, 134, 299-303. [CrossRef]

30. Ferreira, L.E.; Castro, P.M.; Chagas, A.C.; França, S.C.; Beleboni, R.O. In vitro anthelmintic activity of aqueous leaf extract of Annona muricata L. (Annonaceae) against Haemonchus contortus from sheep. Exp. Parasitol. 2013, 134, 327-332. [CrossRef]

31. Iqbal, Z.; Lateef, M.; Ashraf, M.; Jabbar, A. Anthelmintic activity of Artemisia brevifolia in sheep. J. Ethnopharmacol. 2004, 93, 265-268. [CrossRef] [PubMed]

32. Irum, S.; Ahmed, H.; Mirza, B.; Donskow-Łysoniewska, K.; Muhammad, A.; Qayyum, M.; Simsek, S. In vitro and in vivo anthelmintic activity of extracts from Artemisia parviflora and A. sieversiana. Helminthologia 2017, 54, 218-224. [CrossRef]

33. Borgsteede, H.M.; Couwenberg, T. Changes in LC50 in an in vitro egg development assay during the patent period of Haemonchus contortus in sheep. Res. Vet. Sci. 1987, 42, 413-414. [CrossRef]

34. Akkari, H.; B'chir, F.; Hajaji, S.; Rekik, M.; Sebai, E.; Hamza, H.; Darghouth, M.A.; Gharbi, M. Potential anthelmintic effect of Capparis spinose (Capparidaceae) as related to its polyphenolic content and antioxidant activity. Vet. Med. Czech. 2016, 61, 308-316. [CrossRef]

35. Spiegler, V.; Liebau, E.; Hensel, A. Medicinal plant extracts and plant-derived polyphenols with anthelmintic activity against intestinal nematodes. Nat. Prod. Rep. 2017, 34, 627-643. [CrossRef] [PubMed]

36. Brusotti, G.; Cesari, I.; Dentamaro, A.; Caccialanza, G.; Massolini, G. Isolation and characterization of bioactive compounds from plant resources: The role of analysis in the ethnopharmacological approach. J. Pharm. Biomed. Anal. 2014, 87, 218-228. [CrossRef] [PubMed] 
37. Klongsiriwet, C.; Quijada, J.; Williams, A.R.; Mueller-Harvey, I.; Williamson, E.M.; Hoste, H. Synergistic inhibition of Haemonchus contortus exsheathment by flavonoid monomers and condensed tannins. Int. J. Parasitol. Drugs Drug Resist. 2015, 5, 127-134. [CrossRef] [PubMed]

38. Xutian, S.; Zhang, J.; Louise, W. New exploration and understanding of traditional Chinese medicine. Am. J. Chinese Med. 2009, 37, 411-426.

39. Long, F.; Yang, H.; Xu, Y.; Hao, H.; Li, P. A strategy for the identification of combinatorial bioactive compounds contributing to the holistic effect of herbal medicines. Sci. Rep. 2015, 5, 12361. [CrossRef]

40. Váradyová, Z.; Mravčáková, D.; Holodová, M.; Grešáková, L'.; Pisarčíková, J.; Barszcz, M.; Taciak, M.; Tuśnio, A.; Kišidayová, S.; Čobanová, K. Modulation of ruminal and intestinal fermentation by medicinal plants and zinc from different sources. J. Anim. Physiol. Anim. Nutr. (Berl) 2018, 102, 1131-1145.

41. Gaillard, B.D.; Richards, G.N. Presence of soluble lignin-carbohydrate complexes in the bovine rumen. Carbohydr. Res. 1975, 42, 135-145. [CrossRef]

42. Pisulewski, P.M.; Okorie, A.U.; Buttery, P.J.; Haresign, W.; Lewis, D. Ammonia concentration and protein synthesis in the rumen. J. Sci. Food Agric. 1981, 32, 759-766. [CrossRef] [PubMed]

43. Tufarelli, V.; Casalino, E.; D'Alessandro, A.G.; Laudadio, V. Dietary phenolic compounds: Biochemistry, metabolism and significance in animal and human health. Curr. Drug Metab. 2017, 18, 905-913. [CrossRef] [PubMed]

44. Mavrot, F.; Hertzberg, H.; Torgerson, P. Effect of gastro-intestinal nematode infection on sheep performance: A systematic review and meta-analysis. Parasit. Vectors 2015, 8, 557. [CrossRef]

45. Castagna, F.; Palma, E.; Cringoli, G.; Bosco, A.; Nistico, N.; Caligiuri, G.; Britti, D.; Musella, V. Use of complementary natural feed for gastrointestinal nematodes control in sheep: Effectiveness and benefits for animals. Animals 2019, 9, 1037. [CrossRef]

(C) 2020 by the authors. Licensee MDPI, Basel, Switzerland. This article is an open access article distributed under the terms and conditions of the Creative Commons Attribution (CC BY) license (http://creativecommons.org/licenses/by/4.0/). 